\title{
Resolución de problemas con tecnología en un ambiente de aprendizaje colaborativo wiki en la educación media
}

\author{
Problem solving with technology in a wiki-supported collaborative learning \\ environment in secondary education
}

\section{Resolução de problemas com a tecnologia em um ambiente de aprendizagem colaborativa wiki no ensino médio}

\author{
Gerzon Yair Calle-Álvareza," | Iván Darío Agudelo-Correa ${ }^{\mathrm{b}}$ \\ ${ }^{a}$ https://orcid.org/0000-0002-4083-605I Universidad de Antioquia, Medellín, Colombia \\ b Institución Educativa San José del Citará, Ciudad Bolívar, Colombia
}

- Fecha de recepción: 2019/04/II

- Fecha concepto de evaluación: 2019/10/23

- Fecha de aprobación: 2019/10/28 http://dx.doi.org/ | 0.22335/rlct.v I I i2.876
Para citar este artículo / To reference this article / Para citar este artigo: CalleÁlvarez, Y. G., \& Agudelo-Correa, I. D. (2019). Resolución de problemas con tecnología en un ambiente de aprendizaje colaborativo wiki en la educación media. Revista Logos Ciencia \& Tecnología, I I (2), I5I-I65. http://dx.doi.org/I0.22335/rlct.v I Ii2.876

\section{RESUMEN}

El estudio tiene como finalidad caracterizar el procedimiento que realizan los estudiantes de la educación media para resolver problemas con tecnología en un ambiente de aprendizaje colaborativo apoyado en la wiki. Como método de investigación se utilizó la investigación-acción. Participaron diez estudiantes del grado once, de una institución educativa pública del municipio de Ciudad Bolívar, Antioquia, Colombia. Los resultados evidenciaron que el reconocimiento del contexto es necesario para plantear hipótesis de solución. En las conclusiones se afirma que cuando se aplican las etapas de identificación del problema, búsqueda y selección de información, formulación de hipótesis, ejecución de la hipótesis y evaluación de la solución, los estudiantes tienen mayores posibilidades de encontrar una solución que responda a la problemática inicial.

Palabras clave: aprendizaje colaborativo, educación media, resolución de problemas, tecnologías de la información y la comunicación, wiki 


\section{SUMMARY}

The purpose of the study is to characterize the procedure performed by high school students to solve problems using technology in a collaborative wiki-supported learning environment. Action research was used as the methodology. Ten eleventh-grade students from a public educational institution in the municipality of Ciudad Bolivar, Antioquia, Colombia, participated. The results show that recognition of the context is necessary to propose hypotheses for a solution. The conclusions affirm that when students apply the stages of problem identification, information research and selection, hypotheses formulation, hypothesis execution, and solution assessment, they are more likely to find a solution that responds to the initial problem.

Keywords: Collaborative learning, media education, problem solving, information and communication technologies, wiki

\section{SUMÁRIO}

O estudo tem como objetivo caracterizar o procedimento que os alunos do ensino médio realizam para resolver problemas com a tecnologia em um ambiente de aprendizagem colaborativa suportada no wiki. Como método de pesquisa, foi usada a pesquisa-ação. Participaram dez alunos do décimo primeiro ano, de uma instituição pública de ensino do município de Ciudad Bolívar, Antioquia, Colômbia. Os resultados mostraram que o reconhecimento de contexto é necessário para propor uma hipótese de solução.As conclusões afirmam que, quando são aplicados os estágios de identificação do problema, busca e seleção de informação, formulação de hipóteses, execução de hipóteses e avaliação da solução, é mais provável que os alunos encontrem uma solução que responda ao problema inicial.

Palavras-chave: Aprendizagem colaborativa, ensino médio, resolução de problemas, tecnologias da informação e comunicação, wiki

Incorporar en el aula de clases estrategias para estimular en los estudiantes habilidades para la resolución de problemas, mediante el aprendizaje colaborativo, obliga al profesor a pensar las herramientas que requiere, los estilos de aprendizaje de los estudiantes, los procesos de evaluación, el uso o no uso de las tecnologías de la información y la comunicación (TIC), hasta el papel del profesor en el proceso de enseñanza y aprendizaje. Además, la tecnología desempeña un papel importante apoyando la pedagogía, y coadyuvando al desarrollo de habilidades para la resolución de problemas. Cabero (2015) dice que facilita "que los alumnos aprendan por sí mismos, solos o en grupos, contestando preguntas y resolviendo problemas con la ayuda, la orientación y la guía de su profesor" (p. 24). Por otra parte, Moreno-Martínez, López-Meneses y Leiva-Olivencia (20/8) plantean que no deben satanizarse las máquinas y las tecnologías debido a sus aportes para el trabajo colaborativo y la resolución de problemas, lo que favorecerá superar la brecha digital.

Es necesario que el estudiante fortalezca habilidades que le permitan resolver problemas individual y colaborativamente, y que la escuela cree nuevos escenarios para transformar las formas de enseñar $y$ de aprender, y como afirma Marchesi y Díaz (2009) que tenga "en cuenta la cultura de las nuevas generaciones para que el proceso de enseñanza y de aprendizaje tenga sentido también para los estudiantes y no sea vivido como algo aburrido y ajeno a sus preocupaciones e intereses" (p. 3). Solucionar problemas en un ambiente de aprendizaje colaborativo promueve la escucha del otro, la exposición de ideas, la discusión, el consenso y, posteriormente, el planteamiento de hipótesis. Enseñar y aprender en ambientes colaborativos suscita espacios en los que se desarrollan habilidades donde cada uno es autónomo en su proceso de construcción de conocimiento. Estos ambientes generan múltiples posibilidades y oportunidades de intercambio de aprendizajes, lo que potencia el crecimiento del individuo y del grupo en general, que es el fin del trabajo colaborativo, y se logra estableciendo estrategias didácticas que ayuden con este proceso.

Los resultados de las pruebas del Programa Internacional para la Evaluación de Estudiantes PISA 2015, publicados por la Organización para la Cooperación y el Desarrollo 
Económicos (OCDE, 20I7), colocan a Colombia en los últimos puestos en lo relacionado con la evaluación de resolución colaborativa de problemas de la vida real con un promedio de 429 puntos, muy por debajo de la media (500 puntos) de la OCDE. Cerca del $58 \%$ de los estudiantes colombianos, que presentaron la prueba, obtuvieron un rendimiento por debajo del nivel I (bajo) y menos del I\% de los estudiantes colombianos alcanzaron el nivel 4 (superior).

En el plano colombiano, el Gobierno Nacional propone desafíos para que los profesores incluyan la solución de problemas en su práctica educativa. Es así como el Ministerio de Educación Nacional (MEN, 2008) entre los alcances de la alfabetización en tecnología orienta a propiciar en los estudiantes "el reconocimiento de diferentes estrategias de aproximación a la solución de problemas con tecnología, tales como el diseño, la innovación, la detección de fallas y la investigación" (p. 12).Además, invita a que los estudiantes adquieran habilidades para solucionar problemas que transformen el entorno, que asimilen conceptos de diseño, sistemas tecnológicos, fuentes de energía, procesos productivos, la simulación y el trabajo colaborativo que son otras de las múltiples posibilidades de la enseñanza en tecnología e informática.

Serrano, Cristancho y Soler (2016) llevaron a cabo una investigación con 18 estudiantes de grado décimo de la especialidad de Dibujo Técnico, del Instituto Técnico Industrial Francisco José de Caldas, en Bogotá, Colombia, con los que buscó proponer solución a un problema de diseño, fundamentado en la construcción de estructuras en espagueti. La propuesta se desarrolló en cuatro etapas: comprender el problema, concebir un plan, ejecutar el plan y revisar los resultados. Las conclusiones destacan que el desarrollo de actividades tecnológicas especiales (ATE) fue una oportunidad práctica que favorece la reflexión y la acción hacia la tecnología. Al llevar a cabo la experiencia diseñada, los estudiantes se enfrentaron a situaciones que exigieron la comprensión de principios teóricos y reflejar sus aprendizajes para construir conocimientos relacionales y funcionales; y que los objetivos, acciones y resultados atienden al mismo propósito, permitiendo el enfoque profundo de aprendizaje de la tecnología.

Este artículo tiene como objetivo caracterizar el procedimiento que realizan los estudiantes de la educación media para resolver problemas con tecnología en un ambiente de aprendizaje colaborativo apoyado en la wiki.Vale aclarar que se plantea la resolución de problemas con tecnología como un sinónimo de solución de problemas con tecnología, para atender a uno de los cuatro componentes de los estándares de tecnología e informática que redactó el MEN (2008) y que los nombra en el siguiente orden: naturaleza y evolución de la tecnología, apropiación y uso de la tecnología, solución de problemas con tecnología, y tecnología y sociedad. De acuerdo con el MEN (2008), la resolución de problemas con tecnología

se refiere al manejo de estrategias en y para la identificación, formulación y solución de problemas con tecnología, así como para la jerarquización y comunicación de ideas. Comprende estrategias que van desde la detección de fallas y necesidades, hasta llegar al diseño y a su evaluación (p. 14).

Con frecuencia, los profesores de las instituciones educativas dedican su cátedra a enseñar a manejar herramientas ofimáticas (Word, Excel y PowerPoint), el Internet para hacer búsquedas y en ocasiones el uso de blogs o presentaciones en línea con el empleo de Prezi. Sin embargo, la competencia "resolución de problemas con tecnología" como lo expresa el MEN (2008) en su documento "Orientaciones generales para la educación en tecnología", pocas veces se desarrolla con los estudiantes, porque es difícil de abordar, requiere preparación y cambiar el rol de profesores y estudiantes, donde el estudiante es el actor principal, el artífice de su conocimiento y el profesor deja de ser el protagonista, se convierte en un facilitador $y$ copartícipe de los procesos de aprendizaje junto con sus estudiantes. Por ello, la presente investigación pretende ser un aporte para continuar estudiando estrategias que conduzcan a la resolución de problemas con o sin tecnología, estrategias que lleven al estudiante a ser más crítico, exponer sus ideas, discutir, argumentar, concluir y solucionar problemas de la vida diaria.

\section{Marco teórico}

Existe un problema cuando se encuentra una diferencia entre la situación actual y la situación que se considera ideal, y para dar respuesta se apela a conocimientos diversos en los que se utilizan la lógica, las técnicas y las herramientas que se aplican en forma organizada. Poblete y Villa (2007) sostienen que

un problema es una cuestión que no tiene una solución predefinida. El problema, además, tiene que ser una cuestión interesante, que provoque las ganas de resolverla, una tarea a la que se esté dispuesto a dedicarle tiempo y esfuerzos (p. 139). 
Si se resuelve satisfactoriamente, provoca una sensación de agrado y motivación para desarrollar nuevos retos.

De acuerdo con Pozner y Fernández (2000), “los problemas nacen de un malestar, de la identificación de una dificultad o del entorpecimiento de una aspiración o necesidad" (p. 13). Los problemas surgen al tiempo en que se hace presente una necesidad humana, es allí cuando las personas, con el ánimo de superar esa precariedad, intentan dar solución a ese problema acudiendo a su ingenio, destreza, información y conocimientos previos. En esta línea, Gutiérrez-Rodríguez (2018) plantea que las competencias de interpretación y solución de problemas deben promoverse en la escuela por su importancia para responder a los retos que se les presentan a las personas a lo largo de la vida.

La OCDE (2017) define la resolución colaborativa de problemas como

la capacidad de una persona para implicarse de forma eficaz en un proceso en el que dos o más participantes intentan resolver un problema compartiendo la comprensión y el esfuerzo necesarios para llegar a una solución y mancomunar conocimientos, destrezas y esfuerzos para este fin (p. 28).

Este concepto pone de manifiesto la importancia que tiene aplicar los conocimientos adquiridos en otras circunstancias, tanto dentro como fuera de la escuela y en colaboración con otros.

En distintas asignaturas, los estudiantes se ven enfrentados a problemas de distinta naturaleza que requiere de destrezas para resolverlos de la mejor forma posible. Solano (2012) afirma que "la resolución de problemas permite a los estudiantes involucrarse en procesos cognoscitivos superiores como visualización, asociación, abstracción, comprensión, razonamiento, análisis, síntesis y generalización" (p. 32), que son habilidades que permiten que el estudiante adquiera un pensamiento crítico y reflexivo. A su vez, Coronel y Curoto (2008) sostienen que "la enseñanza desde esta perspectiva pretende poner el acento en actividades que plantean situaciones problemáticas cuya resolución requiere analizar, descubrir, elaborar hipótesis, confrontar, reflexionar, argumentar y comunicar ideas" (p.464). Por tanto, es necesario disponer los estudiantes para trabajar en este modelo, teniendo en cuenta los diferentes procedimientos a seguir para resolver problemas de la escuela que luego pueden replicarse a problemas de la vida diaria.

Algunos autores proponen secuencias de pasos para solucionar problemas, entre ellos Bransford y Stein (1986), que consideran la resolución de problemas como un proceso que incluye seis pasos: identificar el problema, definir y representar el problema, explorar posibles estrategias, descomponer el problema en sus componentes elementales, actuar según las estrategias y evaluar y examinar los logros. A su vez, Polya (1989), en su libro "Cómo plantear y resolver problemas", propone que para resolver cualquier tipo de problema se debe comprender el problema, concebir un plan, ejecutar el plan y examinar la solución.

Atendiendo a la función que cumplen los procedimientos o estrategias para la solución de un problema, se podría diferenciar cinco tipos de procedimientos, según Pozo y Postigo (1993):“I) adquisición de la información, 2) interpretación de la información, 3) análisis de la información y realización de inferencias, 4) comprensión y organización conceptual de la información y 5) comunicación de la información" (p. 10). Esto no quiere decir que toda resolución de problemas tenga que ser sometido estos cinco pasos, que en algunos casos pueden ser difusos, 0 que se deban seguir en ese orden secuencial, porque cada problema requiere un enfoque diferente para su mejor comprensión.

A su vez, Pozner y Fernández (2000) proponen las siguientes etapas: I) identificar el problema, 2) explicar el problema, 3) idear las estrategias alternativas, 4) decidir la estrategia, 5) diseñar la intervención, 6) desarrollar la intervención y 7) evaluar los logros. La metodología, de acuerdo con las autoras, hace que se transite por estas siete etapas en forma sucesiva, pero con la posibilidad de retroceder a las etapas anteriores para afianzar conocimientos y poder avanzar con mayor decisión.

Peralta (2018) sostiene que uno de los elementos fundamentales para la formación académica consiste en la enseñanza basada en la resolución de problemas y que los métodos tradicionales se enfocaron en transmitir al aprendiz una heurística determinada sobre la manera en la que se resuelven problemas, pero dicho método no promueve una comprensión compleja del problema, lo que limita al solucionador una escasa capacidad de acción. Este autor hace un estudio de algunas estrategias, como lo es el caso de Yahoo Respuestas, el fenómeno de los 
videoblogs, el uso de la hipermedia, entre otros. Finalmente, se propone la teoría de la actividad histórico-cultural (CHAT) como paradigma capaz de abonar procedimientos y fundamentos sobre la resolución de problemas.

La resolución de problemas planteada como una estrategia educativa, invita a profesores y estudiantes a explorar nuevos ámbitos de conocimiento. Navarro (2006) expone:

Esta estrategia acerca a los alumnos a la identificación del problema, la formulación de predicciones e hipótesis, la relación entre variables, el diseño experimental, la observación, medición, clasificación, seriación y demás técnicas de investigación, transformación e interpretación y análisis de datos, la utilización de modelos, la elaboración de conclusiones, el desarrollo de destrezas manuales y de comunicación (p. 62).

La resolución de problemas con o sin tecnología permite que los estudiantes desarrollen habilidades para identificar problemas, hallar la información requerida, buscar posibles soluciones y evaluar si esas soluciones encontradas si son las adecuadas para el problema planteado inicialmente. Para ello, Morales y Landa (2004) sugieren a los profesores que se trabaje en grupos o equipos pequeños para que haya una mayor interacción y participación de los estudiantes $y$, además, se propongan problemas que sean del interés de los estudiantes, de su cotidianidad para captar su interés y ganas de buscar una solución. A su vez, Calle (2013) plantea que el uso de tecnologías digitales aportará para la comunicación efectiva durante la resolución de problemas por parte de los estudiantes.

\section{Metodología}

El estudio se desarrolló desde las orientaciones de la investigación-acción. De acuerdo con McKernan (1999), "la investigación-acción la llevan a cabo los profesionales en ejercicio tratando de mejorar su comprensión de los acontecimientos, las situaciones y los problemas para aumentar la efectividad de su práctica" (p. 24). Este método "supone entender la enseñanza como un proceso de investigación, un proceso de continua búsqueda" (Bauselas, 2004, p. I). Además, como afirma Tejedor (2018), "la investigación en la acción pretende contribuir tanto a los intereses prácticos de las personas como a los objetivos de la ciencia social" (p. 317).

Rodríguez y Valldeoriola (20I2) plantean que "la investigación-acción se orienta hacia la resolución de problemas mediante un proceso cíclico que va desde la actividad reflexiva a la actividad transformadora" (p. 63). En la misma línea, Torrecilla (2010) considera que "estas actividades tienen en común la identificación de estrategias de acción que son implementadas y más tarde sometidas a observación, reflexión y cambio" (p. 3). El método consiste en que los profesores y estudiantes realicen una serie de actividades, con el fin de optimizar los procesos, mejorar y construir dispositivos en forma colaborativa que den solución a problemas cotidianos.

\section{Etapas}

A continuación, una breve descripción de las actividades que se desarrollaron siguiendo las etapas propuestas por Evans (20I0):

Planteamiento del problema: en esta etapa se realizó una lectura de los documentos relacionados con las pruebas PISA, leyes educativas colombianas, documentos institucionales y de la teoría sobre la resolución de problemas, el trabajo colaborativo y el uso de la wiki en la educación. Al final, se plantearon una pregunta y un objetivo de investigación.

Hipótesis de acción y plan de acción: luego de plantear la pregunta de investigación, se identificó una solución viable a dicha pregunta y para ello se consultaron algunos teóricos que han escrito sobre resolución de problemas, trabajo colaborativo y el uso de la wiki en la educación. Se diseñó el ambiente de aprendizaje colaborativo apoyado en la wiki. Al respecto, Calle (20l3) afirma que

estos espacios se diseñan para crear condiciones pedagógicas que favorezcan el aprendizaje y que responda a las necesidades del contexto educativo, donde los profesores y estudiantes siguen siendo partícipes, el primero como orientador, y el segundo como sujeto activo de su propio aprendizaje (p. 2).

También en esta etapa se eligió una metodología, el enfoque y los instrumentos que dieron evidencia de los resultados. Luego, se convocaron los estudiantes, se citó a reunión con sus padres o acudientes y a los directivos docentes para presentar el proyecto, los compromisos institucionales y solicitar los permisos necesarios para la participación de los estudiantes.

Desarrollo de la propuesta de mejoramiento: en esta etapa se plantearon tres situaciones problemas 
(desechos tecnológicos, página web y aplicación móvil) que debían ser solucionados en forma colaborativa y apoyados por una wiki en cinco pasos (identificación del problema, búsqueda y selección de información, formulación de hipótesis tendientes a la solución, ejecución de la solución y evaluación de la solución). Al finalizar cada situación problema se evaluó el producto obtenido con instrumentos como un protocolo escrito de verbalización retrospectiva para los desechos tecnológicos, coevaluación para la página web y matriz de valoración para la aplicación móvil.

Durante la implementación de esta propuesta se utilizó la wiki como un espacio de interacción, de debate, socialización y repositorio de información donde se vieron reflejadas todas y cada una de las etapas de la resolución de problemas. La intervención se llevó a cabo en veinte semanas, en las que los estudiantes asistieron a la sala de sistemas de la Institución Educativa San José del Citará, durante cuatro horas semanales. La sala de sistemas estaba dotada con 30 computadores de escritorio y 6 computadores portátiles, un video beam, un tablero acrílico y banda ancha de $30 \mathrm{Mb}$.

Evaluación y lecciones aprendidas: en esta etapa se analizaron los instrumentos considerados al comienzo de la investigación de la entrevista semiestructura y el diario de campo. Se consolidaron las respuestas de la entrevista y los apuntes del diario de campo en un archivo de Microsoft Excel, se realizó lectura interpretativa y desde el análisis de contenido se determinaron las relaciones entre las subcategorías que emergían y cada fase de la resolución de problemas. Luego, se trianguló la información obtenida en estos instrumentos y se relacionó con la teoría consultada. Finalmente, se plantearon las conclusiones.

Difusión de resultados: al finalizar la resolución de las situaciones problema se presentó a toda la comunidad educativa de la Institución Educativa San José del Citará, en el caso de la página web, se le hizo una exposición a profesores, estudiantes y padres de familia para que la visitaran y le dieran uso; con el caso de la aplicación se hizo igual y se puso al servicio de los profesores de primaria instalándola en algunas tabletas que podían usar en sus clases.

\section{Población}

La investigación se llevó a cabo en el segundo periodo del 2017, con un grupo de estudiantes del grado once, pertenecientes a la media técnica en informática de la
Institución Educativa San José del Citará del municipio de Ciudad Bolívar, Antioquia. Este grupo estaba conformado por diez estudiantes, entre ellos seis mujeres y cuatro hombres de edades que oscilan entre los 16 y los 20 años.

\section{Instrumentos de investigación}

Entrevista semiestructurada: este instrumento tiene como propósito identificar los procedimientos no visibles que realizan los estudiantes en el proceso de resolución de problemas con tecnología. Esta contenía unas instrucciones para el entrevistador y cuatro preguntas orientadoras con relación a los siguientes ítems: identifica situaciones problema, búsqueda y selección de información, proposición de hipótesis de solución, evaluación de la solución presentada y trabajo en equipo. La entrevista se aplicaba al finalizar cada situación problema a los diez estudiantes.

Diario de campo: el diario de campo fue elaborado por el profesor investigador y aplicado por él mismo durante el proceso de solución de las situaciones problema. Se registraban observaciones generales en la que se incluían los elementos emergentes asociados a la resolución de problemas con tecnología. Para el diligenciamiento de este instrumento, el investigador observaba los estudiantes cómo asumían el rol que les correspondía, en otras ocasiones el profesor dialogaba con ellos para conocer lo que estaban tratando de hacer.

\section{Situaciones problemas}

Situación I - Desechos tecnológicos (SRRAT). En los últimos años se ha multiplicado la presencia de equipos electrónicos en los hogares y oficinas. El problema era identificar una forma de uso de los residuos tecnológicos que ha generado la institución educativa en los últimos tres años. Los estudiantes comprendieron el problema y reconocieron su importancia en la conservación del medioambiente.

Situación 2 - Página web. El docente investigador les sugirió a sus estudiantes la siguiente situación: los propietarios del "Café don Édgar" desean que su tienda sea conocida en la Internet y que sus clientes puedan saber qué productos venden y cuál es su precio, publicar los datos de la empresa y que sus clientes puedan hacer sus pedidos vía web, ¿cómo podrías ayudarles a los propietarios del 
café? Sin embargo, los estudiantes propusieron algunas otras opciones y decidieron finalmente crear una página web relacionada con la celebración, en ese entonces, de los 80 años de su Institución Educativa San José del Citará. Vale anotar, que para ese momento, la institución no contaba con página web.

Situación 3 - Aplicación Móvil (ABCrea). El docente investigador le propuso a sus estudiantes crear una aplicación que diera solución al siguiente problema: los estudiantes conocen el sistema de evaluación de la Institución Educativa San José del Citará y saben que los dos primeros periodos académicos tienen una valoración del $20 \%$ cada uno y los dos últimos periodos del $30 \%$ cada uno, pero no saben cómo calcular la nota que requieren en cada uno de los periodos, después del primer periodo académico, para aspirar a ganar una asignatura al finalizar el año. Con una aplicación instalada en un dispositivo electrónico como Smartphone, Tablet o computador podrán calcular esas notas de una manera fácil y cómoda. Sin embargo, los estudiantes objetaron la propuesta y discutieron otras opciones, finalmente se pusieron de acuerdo en crear una aplicación que ayudara a los estudiantes de primaria en el proceso de lectoescritura, debido a la importancia de esta asignatura en la formación académica.
Modelo para la resolución de problema con tecnología

Para esta investigación en particular, se tuvo en cuenta las propuestas de Bransford y Stein (1986) y Polya (1989) con algunos leves cambios como puede apreciarse en la figura I. Se hizo en cinco fases que quedaron registradas por los estudiantes en una wiki, a medida que ellos fueron avanzando. Estas fases estuvieron mediadas por la wiki y se llevaron a cabo en el siguiente orden: identificación del problema, búsqueda y selección de información, formulación de hipótesis tendientes a la solución, ejecución del plan de solución y evaluación de la solución.

\section{Resultados y discusión}

Para llevar a cabo la resolución de problemas con tecnología se usó un modelo obtenido de la mezcla de las propuestas de Bransford y Stein (1986) y Polya (1989), que el autor de esta investigación resumió en cinco etapas: identificación del problema, búsqueda y selección de información, formulación de hipótesis tendientes a la solución, ejecución de la hipótesis y evaluación de la solución. A continuación, se presentan los resultados según cada una de las etapas:

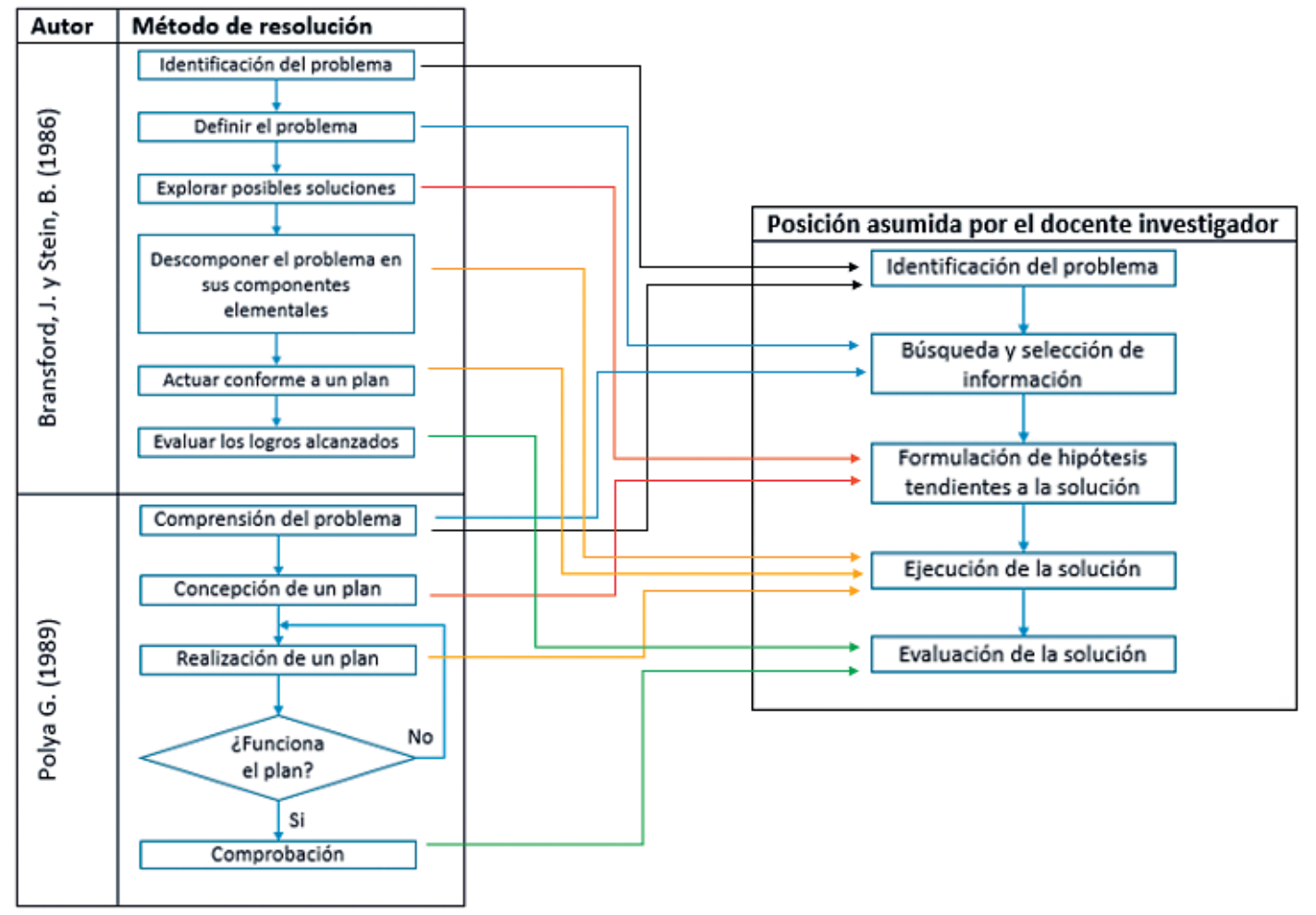

Figura I. Métodos de resolución de problemas y posición asumida por el docente investigador 


\section{Identifica situaciones problema}

En la entrevista semiestructura se plantearon tres preguntas orientadoras a la identificación de situaciones problemas: ¿Trabajar en equipo te ha sido útil para identificar problemas? ¿Cuáles indicios de los planteamientos presentados te ayudaron a identificar el problema? ¿Qué elementos se deben considerar al momento de identificar una situación problema? Las respuestas evidenciaron que el $80 \%$ de los estudiantes respondió que el trabajar en equipo favorece la comprensión de situaciones problemas. El $80 \%$ de los estudiantes indicó que decidieron investigar problemas que le beneficien o estén relacionados con la institución educativa. El $80 \%$ manifestaron que es importante tener en cuenta el entorno y la población que puede beneficiarse al momento de analizar las situaciones problemas.

Analizadas las tres preguntas relacionadas con identificar situaciones problema y los registros del diario de campo, se identificó que los estudiantes cuando trabajan en equipo reconocen situaciones problema con mayor facilidad y eligen temas que beneficien a la población tanto del colegio como del entorno cercano a su hogar. Una estudiante planteó:"el trabajo en equipo fomenta la cooperación, allí analizamos los puntos de vista de cada compañero para de esta forma llegar a un acuerdo, el cual nos lleva a una solución". Este concepto lo corroboran Revelo-Sánchez, Collazos-Ordóñez y Jiménez-Toledo (2018), cuando afirman que algunas características del trabajo colaborativo son "la negociación y los procesos de diálogo que se dan al interior del grupo" (p. 3). Igualmente, Echazarreta, Prados, Poch y Soler (2009) indican que "el trabajo colaborativo exige a los participantes habilidades comunicativas, relaciones simétricas y recíprocas, así como un deseo de compartir la resolución de las tareas” (p. 4).

Durante la fase de identificación del problema, las orientaciones dadas por el profesor eran muy importantes. Según Travieso-Valdés y Ortiz-Cárdenas (2018), la primera etapa de la resolución exige que el profesor sea lo más claro y preciso con la información asociada al problema. Después de la exposición del problema por parte del profesor, los estudiantes se plantearon y resolvieron preguntas sobre el contexto, las razones y posibles consecuencias del mismo. Esto condujo a los estudiantes a debatir con argumentos cada una de las posibles situaciones que iban a resolver, finalmente, llegaron a acuerdos para seleccionar un problema al cual le apuntarían para encontrar una solución. En el caso SRRAT, fue fácil que los estudiantes estuvieran de acuerdo; con el caso de la página web, hubo gran controversia, debido a que la mayoría de los estudiantes no querían hacer una página web para una empresa privada y finalmente decidieron hacer la página para la Institución Educativa San José del Citará; con el caso de la aplicación móvil ABCrea, los estudiantes a través de una lluvia de ideas, decidieron hacer una aplicación móvil que ayudara a los estudiantes de primaria en la comprensión lectora, la inferencia y el reconocimiento del abecedario.

\section{Busca y selecciona información}

En la entrevista semiestructurada se hicieron cuatro preguntas sobre la forma en que los estudiantes buscaban y seleccionaban la información: ¿Qué medios utilizaste para la búsqueda de información? ¿Cómo hizo el equipo para seleccionar la información más apropiada? ¿Cuáles estrategias de clasificación utilizaban para la selección de información? ¿La información montada en la wiki les ayudó a solucionar los problemas? Las respuestas arrojaron que el $100 \%$ de los estudiantes manifestaron que usaron las herramientas como Google para hacer sus búsquedas, también realizaban entrevista a personas externas e internas a la institución sobre la temática, por ejemplo, profesores y familiares. El $50 \%$ de los estudiantes indicaron que entre todos se acordó cuál era la información más relevante. El $70 \%$ de los estudiantes estuvo de acuerdo en que la clasificación de la información hallada la hicieron en equipo, seleccionando la información más relevante y consecuente. El 100\% manifestaron que la wiki ayuda a la consolidación de la información encontrada y seleccionada.

A partir de las respuestas de los estudiantes y la observación realizada por el investigador, puede decirse que los estudiantes acudieron con frecuencia a Internet, subieron la información a la wiki, que fue de gran ayuda y entre todos seleccionaron la información más relevante y consecuente. Una estudiante expresó: "Se buscó información que nos sirviera para llevar a cabo nuestro trabajo, después cada uno socializó lo que había consultado y entre todos seleccionamos la que estuviera mejor". Es evidente que la web 2.0 tiene un papel importante, pero por encima de esto el trabajo colaborativo contribuye a solucionar problemas de la vida cotidiana, tal como lo afirma Solano (2012) cuando dice que "la Wiki, como entorno de interacción y construcción colaborativa, permite evidenciar el trabajo en grupo y aprendizaje colaborativo, que son prácticas de la vida cotidiana” (p. 21). Igualmente, GutiérrezRodríguez (2018) plantea que la escuela debe aportar la formación de competencias en la resolución de problemas. 
Durante la fase de búsqueda de información, los estudiantes usaron varias fuentes de información como es el caso de entrevista a profesores, estudiantes, personas externas al colegio e Internet, en todos los casos haciendo búsquedas en Google, leyendo tutoriales y videos de YouTube. En el caso SRRAT, los estudiantes hicieron llamadas a empresas de las ciudades de Medellín y Bogotá para indagar sobre la posibilidad de recolección de desechos tecnológicos o reciclaje de los mismos. En el caso de la página web institucional, los estudiantes indagaron con profesores, la secretaría, coordinación y rectoría del colegio en busca de la información que debía contener la página web, también se hizo una búsqueda en Internet de las mejores plataformas que había en el momento para crear la página. En el caso de la aplicación móvil ABCrea fue de gran importancia entrevistar a las profesoras que en ese tiempo daban clase en los grados de primaria para identificar los requisitos y las necesidades que ellas percibían en sus estudiantes, igualmente los estudiantes recurrieron al uso de tutoriales y videos explicativos para construir el código de la aplicación.

\section{Propone hipótesis de solución}

En la entrevista semiestructurada se plantearon cuatro preguntas sobre la proposición de hipótesis de solución: ¿Cuál fue el procedimiento que utilizaste para determinar una hipótesis de solución a las situaciones problemas con tecnología? ¿Las hipótesis que aportaste fueron acogidas por el grupo o no? ¿Crees que las hipótesis aportadas por tus compañeros son valiosas? ¿Cómo llegaron a acuerdos en la hipótesis final? Las respuestas permitieron identificar que el $40 \%$ de los estudiantes indicaron que para determinar la hipótesis tuvieron en cuenta las necesidades del colegio. El $30 \%$ indicó que tuvieron en cuenta las opiniones y sugerencias de los docentes. El 100\% de los estudiantes estuvieron de acuerdo en que las hipótesis planteadas por sus compañeros eran valiosas, se tenían en cuentas sus opiniones y entre todos crearon una hipótesis más coherente. El 100\% coincidieron en que llegaron a acuerdos a través de la exposición de sus ideas $y$, finalmente, construyeron la hipótesis por decisión de grupo.

A partir de las respuestas de la entrevista semiestructurada y los registros del diario de campo relacionadas con proponer hipótesis de solución, puede decirse que los estudiantes manifestaron que las hipótesis planteadas por ellos eran valiosas y respetadas por los compañeros del grupo, pero que finalmente elegían entre todos, una hipótesis grupal que respondiera a las condiciones del entorno donde se pretendía aplicar. Esto concuerda con Calzadilla (2002), quien asegura que

en el aprendizaje colaborativo... cada uno comprende la necesidad de aportar lo mejor de sí al grupo para lograr un resultado sinérgico, al que ninguno accedería por sus propios medios; se logra así una relación de interdependencia que favorece los procesos individuales de crecimiento y desarrollo, las relaciones interpersonales y la productividad (p. 4).

Una estudiante expresó que "todo el grupo dio argumentos de lo que habíamos visto del problema y cada uno dio soluciones... muchas de ellas coincidían... por esa razón no hubo tanto problema para tener acuerdos en la hipótesis final”.

Para la fase de formulación de hipótesis, los estudiantes por equipos de trabajo colaborativo proponían una posible hipótesis de solución a cada caso, la exponían, la argumentaban y discutían, finalmente la hipótesis mejor argumentada o con mayor número de votos, era la que elegían para continuar el proceso de resolución de problemas. En el caso SRRAT, los estudiantes acordaron recuperar aquellos computadores antiguos, instalándole un sistema operativo Linux liviano y ponerlo al servicio de la biblioteca para que los estudiantes hagan consultas. También se hicieron contactos con empresas recicladoras para que se acercaran al colegio a recoger aquellos equipos que no podían ser recuperados en el colegio. En el caso de la página web institucional, se acordó usar la plataforma Webnode, porque no mostraba publicidad en las páginas, tenía múltiples plantillas y podían usarla por periodos largos de tiempo. En el caso de la aplicación móvil $A B C r e a$, los estudiantes decidieron que trabajarían App Inventor porque los estudiantes la habían usado anteriormente en el programa InnovaLab de la Gobernación de Antioquia $y$, por tanto, se sentían familiarizados con esta herramienta.

\section{Ejecución de hipótesis}

La ejecución de la hipótesis, de acuerdo con el concepto de los estudiantes involucrados en este proyecto, fue la fase más difícil. Organizados por equipos de trabajo colaborativo y de acuerdo con sus roles, los estudiantes llevaban a cabo aquello que había acordado en la fase anterior, repartían el trabajo de acuerdo con las habilidades de cada uno, así por ejemplo los estudiantes más hábiles para dibujar se encargaban de los diseños, quienes eran buenos en la redacción escribían en la wiki y los que mejor 
programaban se dedicaban a la codificación en la página web o en la aplicación móvil. Es importante anotar que se debía elegir con mucho cuidado una hipótesis coherente para no tener que devolverse de esta etapa a la primera y tener que volver a empezar todo desde el principio, pero que no deja de ser un tiempo de aprendizaje para el estudiante, porque el ensayo error también es un método para resolver problemas; al respecto, Travieso-Valdés y Ortiz-Cárdenas (2018) plantean que el error se convierte en la base del aprendizaje.

\section{Evalúa la solución presentada}

Las preguntas que se plantearon a los estudiantes fueron: ¿Cuáles fueron los criterios que determinaron para decidir que la solución al problema con uso de tecnología podría ser efectiva? ¿Cuáles dificultades se presentaron durante el proceso de solución de problemas con tecnología? ¿De las etapas para solucionar problemas, consideras que algunas son más difíciles que otras? El $40 \%$ de los estudiantes consideraron que el reconocimiento de la población donde se aplicaría la resolución era un indicador para determinar la solución adecuada. Además, el $20 \%$ consideró la importancia de las fuentes de consulta y los mecanismos de solución. El $40 \%$ de los estudiantes respondió que la ejecución es la más difícil, porque ahí es donde surgen los problemas; el $20 \%$ dijo que la etapa más compleja fue la formulación de la hipótesis.

Para la evaluación de la propuesta de la solución del problema, los estudiantes determinaron cuatro criterios iniciales: que la información fuera verídica, que se hubiera respetado los pasos para hallar la solución, los acuerdos de grupo y población beneficiaria. Lo que demuestra cómo los estudiantes aplicaron estrategias colaborativas para evaluar los aciertos o desaciertos al momento de implementar una solución, y que esta respondiera a la realidad institucional. Lo anterior concuerda con la afirmación de Escudero (2009) sobre las estrategias de problematización y acuerdos del grupo de trabajo, lo que aporta al desarrollo cognitivo para la resolución de problemas apoyados por las TIC.

A la hora de evaluar la solución presentada, los estudiantes se refirieron a la aplicación móvil y manifestaron que se inclinan por aquellas soluciones que fueran más atractivas para los niños, aunque esto les generó dificultades en la programación y reconocieron que faltó comunicación y compromiso por parte de ellos mismos. Además, la mayoría concluyó que de todas las etapas para solucionar problemas la más difícil fue la ejecución de la hipótesis. Los estudiantes cuando evalúan la solución demostraron que para ellos fue importante la apariencia visual y reconocieron que lo actitudinal tiene un papel importante a la hora de solucionar problemas.

Para la evaluación de la solución, el profesor investigador elaboró un instrumento para cada uno de los casos o problemas que requerían una solución. En el caso SRRAT, el profesor aplicó un protocolo escrito de verbalización retrospectiva como sugiere García (1988), en el que los estudiantes manifiestan las experiencias vividas en el proceso de resolución del problema con tecnología en forma colaborativa y apoyados por una wiki. En el caso de la página web institucional, se aplicó una coevaluación y tenía como objetivo analizar del proceso de solución que se llevó a cabo (página web), con el fin de que se retroalimenten y se tengan en cuenta la metodología para futuras soluciones a problemas y de ser posible se verifique si puede obtenerse el mismo resultado de otra manera. En el caso de la aplicación móvil ABCrea, se aplicó una matriz de valoración del programa o aplicación (Vaca, Agudo \& Sánchez, 2014) en la que estudiantes del grado 10 y profesores del área de tecnología e informática calificaron la aplicación, después de observar detenidamente el funcionamiento de la misma.

\section{Trabajo colaborativo}

Las preguntas planteadas sobre el trabajo colaborativo fueron: ¿Qué crees que se debe hacer para que el trabajo colaborativo sea más efectivo? ¿Qué crees que es mejor trabajar de manera colaborativa $\circ$ individual? ¿Crees que vale la pena trabajar en equipo usando una wiki? ¿Es fácil o difícil trabajar en grupo usando una wiki? El $60 \%$ de los estudiantes respondieron que para trabajar en equipo se requiere compromiso, responsabilidad por parte de los estudiantes y establecer tiempos. El $60 \%$ dijo que es mejor trabajar colaborativamente porque todos aportan. El $50 \%$ respondió que la wiki es una buena herramienta para trabajar en equipo a distancia y presencial.

Revisando las respuestas de los estudiantes, asociadas al trabajo colaborativo y los registros del diario de campo, puede decirse que los estudiantes manifestaron que es mejor trabajar colaborativamente con la ayuda de una wiki siempre y cuando haya dedicación, compromiso y responsabilidad de los integrantes del equipo de trabajo. Ellos expresaron que para ellos es importante lo actitudinal, lo técnico y el aporte grupal. Al respecto, Solano 
(20I2) sostiene que la wiki es un "espacio de interacción y colaboración entre los estudiantes para compartir conocimiento, y para ayudarse en la realización de tareas" (p. 228). Igualmente, Calle (20I3) afirma que para que haya trabajo colaborativo es necesaria la confianza y corresponsabilidad entre sus integrantes.

Con relación al trabajo colaborativo, puede inferirse que se sintieron motivados trabajando en equipo porque se respetaban las opiniones de todos, aprendían de sus compañeros y sintieron que sus propuestas eran valiosas, manifestaron el orgullo que sentían con el producto final. Al respecto, un estudiante expresó:

Al principio no tenía clara ciertas cosas, por lo tanto, no aportaba, pero después me focalicé en el tema y aporté positivamente. Con relación al trabajo colaborativo, fue muy enriquecedor trabajar con mis compañeros, escucharlos, compartir y hasta reír con ellos, todo fue muy agradable y para mí, nosotros hicimos el mejor trabajo.

Esto coincide con Maldonado (2008), quien afirma que uno de los beneficios del aprendizaje basado en problemas es que aumenta la autoestima, ya que "los estudiantes se enorgullecen de lograr algo que tenga valor fuera del aula de clase" (p. 163).

Para esta investigación se usó una wiki de Google Site por la facilidad de integrar archivos de Google Drive y efectivamente se logró sincronizar estas herramientas para que el trabajo de los estudiantes fuera más efectivo, de tal manera que los ajustes que hacían ellos en un documento u hoja de cálculo, inmediatamente tenían efectos en el documento que se exponía en la wiki. Los estudiantes compartían los documentos, hojas de cálculo o presentaciones y desde cualquier lugar geográfico aportaban a la creación de ese archivo e inmediatamente se observaba en la wiki. Esto facilitó el trabajo colaborativo en la resolución de problemas para aquellos estudiantes que tenían Internet en sus hogares, pero también es bueno decir que otros que no contaban con tal suerte, no interactuaron de la misma manera y solo lo hacían cuando estaban en el colegio y tenían acceso a un computador con Internet.

\section{Discusión}

La resolución de problemas con tecnología, en ocasiones no es abordada por los profesores porque requiere preparación y cambiar el rol para dejar de ser el protagonista y convertirse en un facilitador y copartícipe con los estudiantes. Para llevar a cabo la resolución de problemas, en esta investigación se hizo una mezcla de las técnicas usadas por Bransford y Stein (1986) y Polya (1989) que pueden resumirse en identificación del problema, búsqueda y selección de información, formulación de hipótesis tendientes a la solución, ejecución de la hipótesis y evaluación de la solución. Al comienzo de la resolución de problemas se pudo evidencias que a los estudiantes les cuesta trabajar en forma colaborativa, pero con el paso del tiempo descubrieron que el aporte de los compañeros los enriquecía a todos, que las habilidades de unos fueron aprovechadas por todo el equipo, entonces mostraron respeto por las ideas de cada uno, dividieron el trabajo, hubo compromiso, dedicación, debates con argumentos y finalmente acuerdos para solucionar el problema planteado. La wiki ayudó como sitio de encuentro, discusión y repositorio de ideas, archivos, imágenes y videos a los que todos podían acceder desde un dispositivo con acceso a Internet.

Se observó que los estudiantes involucrados en este proyecto, en la etapa de identificación del problema, ya estaban haciendo hipótesis y querían pasar de una vez a la etapa de ejecución; en la etapa de selección de información, descubrían que estaban equivocados o corroboraban que iban por el sendero correcto en busca de la solución, pero siempre estuvieron de acuerdo en que la etapa con mayor grado de facultad fue la etapa de ejecución de la hipótesis seleccionada para resolver el problema, porque en ocasiones les obligaba a devolverse y empezar de nuevo el proceso. Con relación al trabajo colaborativo, los estudiantes asignaron roles de acuerdo con las habilidades especiales que los estudiantes tenían y para tomar decisiones exponían sus puntos de vista y finalmente llegaban a acuerdos para encontrar la solución al problema planteado, en concordancia con lo expuesto por Gutiérrez, Yuste, Delgado y Fustes (20lI). La wiki contribuyó a la participación, puesta en común, discusión y repositorio de información de todos y cada uno de los estudiantes en cada una de las etapas para solucionar problemas. A juicio de Solano (20I2), la participación de los estudiantes "evidencia la utilidad de la Wiki como espacio de interacción y colaboración entre los estudiantes para compartir conocimiento, y para ayudarse en la realización de tareas" (p. 228).

El liderazgo fue ejercido por varios estudiantes en los distintos casos planteados en esta investigación. En el primer 
caso llamado "desechos tecnológicos" (SRRAT), el liderazgo no fue muy marcado, al parecer por la falta de confianza en sí mismos para trabajar colaborativamente en la solución de problemas con tecnología; pero en el segundo caso llamado "web institucional", un estudiante marcó la pauta para dirigir a sus compañeros y redactar los acuerdos a que llegaba el equipo en cada una de las etapas tendientes a la solución; en el tercer caso llamado "aplicación móvil” (ABCrea), otro estudiante se puso al mando y organizó los equipos para trabajar en cada uno de los módulos que componían la aplicación y lideró el equipo para hacer el ensamble y los ajustes necesarios para que la aplicación estuviera lista para ponerla al servicio de la comunidad educativa. Este resultado tiene concordancia con lo expuesto por Gutiérrez et al. (201 I), cuando dicen que una de las características imprescindibles del trabajo colaborativo es el "liderazgo compartido por todos los miembros del equipo” (p. 184).

Moreno-Martínez et al. (2018) afirman que el uso didáctico de las tecnologías favorece la resolución de problemas de manera colaborativa. Los estudiantes expresaron su gusto por el trabajo colaborativo y consideraron que el aporte de todos fue muy enriquecedor para crecer como equipo, se sintieron más responsables para llevar a feliz término la actividad emprendida; además, la participación por medio de la wiki aportó a la consolidación del grupo y seguimiento a las etapas en la resolución del problema. Durante las actividades que se desarrollaron en el salón de clase con los estudiantes del grado once, pertenecientes a la media técnica en informática, se resaltan los siguientes aspectos: intercambio de ideas a través de exposiciones para llegar a concertaciones que enriquecían el conocimiento del grupo; la responsabilidad con la tarea asignada a cada uno de los miembros favoreció el desarrollo cognoscitivo del equipo de trabajo colaborativo; con el trabajo hecho por los integrantes de cada equipo se pudo evidenciar que los estudiantes se enseñaban unos a otros, se aceptaban las diferencias y se respetaban las opiniones; los estudiantes con habilidades especiales aportaban en aquello que mejor sabían hacer para contribuir a la calidad del trabajo realizado solucionando problemas.

La wiki les ayudó a recopilar la información, les permitió tener un espacio dónde expresar sus ideas e inquietudes, dónde montar las fotografías de lo que iban desarrollando y trabajar desde sus casas, aunque algunos estudiantes se quejaban porque vivían en el campo y no tenían acceso a Internet, entonces su trabajo se reducía a lo que podían hacer en los encuentros de clase. Por otra parte, los proyectos realizados por el equipo cumplían con satisfacción las necesidades que inicialmente se habían planteado y fueron reconocidos por sus pares y profesores en la institución educativa, de tal manera que se siguen utilizando como es el caso de la página web y la aplicación móvil. Esto como asegura Maldonado (2008), "es una estrategia didáctica que proporciona oportunidades a los estudiantes para persistir en el logro de sus propios intereses y los anima para tomar decisiones sobre cómo encontrar respuestas y solucionar problemas" (p. 176). En los estudiantes se evidenció motivación, entusiasmo y orgullo cuando expresaban satisfacción por los logros que iban obteniendo en el desarrollo de este proyecto. Según Gutiérrez-Rodríguez (2018), el uso de tecnologías para la resolución de problemas resulta motivante para los estudiantes.

La web 2.0 fue valiosa en el proceso de resolución de problemas con tecnología, más específicamente la wiki y los archivos de Google Drive que ayudaron a que se resolvieran problemas en un entorno de trabajo colaborativo permitiendo la construcción, discusión y sinergia de equipo. Solano (2012) afirma que la wiki ayuda recopilar y compartir información, les permite tener un espacio dónde expresar sus ideas e inquietudes y trabajar desde otros espacios diferentes al ambiente escolar. Los archivos de Google Drive se compartían entre los integrantes del grupo para que cada uno pudiera editarlos desde el lugar que prefiriera y seguidamente se montaban en la wiki, lo que facilitaba que todos tuvieran acceso a la misma información en todo momento gracias a la sincronización entre estas herramientas.

Díaz (2008) sostiene que las herramientas web 2.0 son "elementos que permitirían innovar la educación sustentada en las TIC a fin de promover el aprendizaje complejo, la construcción significativa del conocimiento y la educación para la vida" (p. I3). Estas herramientas sirvieron de mediadores en la interacción de los estudiantes con sus compañeros y profesores, en la construcción de conocimiento conjunto, pero como los mismos estudiantes lo manifestaron, siempre y cuando haya dedicación, compromiso y responsabilidad en el desempeño de los roles que se asumen. Es de anotar que una de las dificultades que se presenta en el uso de las herramientas web 2.0, es la poca conectividad que hay en pueblos $y$ veredas, lo que hace 
que los estudiantes no participaran con la misma regularidad y su trabajo se viera limitado a lo que realizaron en los encuentros o clases en el colegio.

\section{Conclusiones}

Herramientas digitales como la wiki permiten la interacción de los participantes tanto en forma sincrónica como asincrónica, facilitan la creación, edición y reescritura de contenidos, de acuerdo con las necesidades y decisiones que puedan surgir en el equipo de trabajo. Siempre es bien acogida en esta herramienta, la posibilidad de revisar el historial y verificar algún contenido que por error o descuido se había perdido. Las wikis son una opción para desarrollar las competencias de trabajo colaborativo, siempre y cuando se asignen roles que los estudiantes asimilen para producir esas sinergias de equipo. Las wikis pueden alentar a los estudiantes a copiar información que no es de su autoría y es ahí donde el docente interviene para motivar a los estudiantes a respetar los derechos de autor y dar los créditos al autor intelectual.

El desarrollo de esta investigación mostró que la resolución de problemas con tecnología en un ambiente de aprendizaje colaborativo wiki con estudiantes de educación media promovió en ellos la motivación hacia la consulta y construcción de conocimientos. Acorde con esto, los estudiantes manifestaron sentirse comprometidos y expresaron satisfacción y orgullo con el trabajo realizado. Además, cuando se aplicaron las etapas: identificación del problema, búsqueda y selección de información, formulación de hipótesis tendientes a la solución, ejecución de la hipótesis y evaluación de la solución, los estudiantes tuvieron mayores posibilidades de encontrar una solución que responda a la problemática inicial.

Las tareas de aprendizaje enfocadas a la resolución de problemas con tecnología, pueden consultarse con los estudiantes, con el fin de que sean de su interés o necesidad en el contexto en que se llevan a cabo. Cuando los estudiantes perciben que los problemas que van a resolver son cercanos a ellos y tienen que ver con su comunidad o su entorno es posible que hagan un esfuerzo mayor por resolverlos. Además, la estrategia de resolución de problemas con tecnología es un proceso cognitivo, didáctico y social vigente, por sus grandes posibilidades de aplicación a lo largo de la vida, en situaciones cotidianas.
Al iniciar el proceso de resolución de problemas con tecnología, se pudo evidenciar que a los estudiantes les cuesta trabajar en forma colaborativa, posiblemente por las estrategias pedagógicas que se llevan a cabo en la escuela que favorecen el trabajo individual y se desconocen las bondades del trabajo colaborativo, en el que los miembros de un equipo se complementan, comparten conocimiento, aportan ideas, discuten y llegan a acuerdos, que son habilidades importantes para la vida diaria y el trabajo futuro.

Durante la ejecución de la etapa "identificación del problema", los estudiantes se atrevían a lanzar hipótesis y querían pasar de una vez a la ejecución de la hipótesis. Sin embargo, con la etapa de "búsqueda y selección de información" descubrían que algunas de sus apresuradas hipótesis no eran tan acertadas o no resolvían el problema planteado inicialmente. En la segunda etapa llamada “búsqueda y selección de información", los equipos de trabajo colaborativo consultaron qué hacer con cada uno de los casos, lo que les confirmaba las ideas iniciales que habían planteado como solución o descubrían que estaban equivocados y con argumentos discutían sobre la información más congruente para dicho caso. En ocasiones se presentaban discusiones con algún estudiante, sobre la forma de resolver el problema, pero con los argumentos dados por sus compañeros, que se hacían con exposiciones y gráficas en algunos casos, se despejaban las dudas y llegaban a acuerdos grupales que luego redactaban y publicaban en la wiki.

En la tercera etapa llamada "formulación de hipótesis" tendientes a la solución, los estudiantes proponían teorías respecto a la solución, que se les facilitó con la etapa de "búsqueda y selección de información", algunas inviables en el contexto educativo, como fue el transporte de los desechos tecnológicos a otras ciudades donde le dieran un tratamiento adecuado, por falta de presupuesto; $y$ otras factibles como las que se entregaron como producto final en cada uno de los casos, a la Institución Educativa San José del Citará del municipio de Ciudad Bolívar en Antioquia. La etapa de "ejecución de la hipótesis" fue considerada por todos los estudiantes como la etapa más difícil y la que más tiempo requería para llevarla a cabo y consideraron que fue muy importante la responsabilidad, el compromiso y la dedicación que le pusieron a la resolución de los problemas planteados. 
Durante el desarrollo de esta investigación, se presentaron algunas dificultades que hicieron que el proceso fuera más lento de lo que inicialmente se había previsto. En el primer semestre de 2017, la institución no contó con el servicio de Internet, lo que dificultó el trabajo en general, sobre todo porque los estudiantes necesitaban el servicio para consultar y hacer sus aportes en la wiki. En el caso SRRAT, se hizo necesario conseguir permisos de las entidades territoriales para darles de baja a los equipos obsoletos que estaban inventariados a nombre del colegio y que reposaban en los libros del almacén de la alcaldía municipal de Ciudad Bolívar. En el caso de la página web institucional fue necesario que toda la comunidad educativa se comprometiera para alimentar la página con contenido actual que fuera de interés general, al igual que conformaran un equipo de trabajo, con estudiantes de distintos grados, para que apoyaran esta labor.

Al terminar este trabajo de investigación se identificaron algunas rutas de trabajo que se podrían tener en cuenta en próximas investigaciones, tales como: (a) hacer estudios que permitan analizar la incidencia de los archivos de Google Drive como espacio para desarrollar trabajo colaborativo resolviendo problemas en cualquier área de conocimiento, con estudiantes de primaria, secundaria, media y educación superior; (b) cómo puede aprovecharse el correo electrónico, o mensajes sms (servicio de mensajes cortos) para resolver problemas, en un área específica, de forma colaborativa, en cualquiera de las etapas escolares y (c) llevar a cabo estudios que determinen la incidencia de las redes sociales, entre ellas, WhatsApp a la hora de desarrollar trabajo colaborativo para resolver problemas de interés general.

\section{Referencias}

Bauselas, E. (2004). La docencia a través de la investigación-acción. Revista Iberoamericana de Educación, 35(I), I-9.

Bransford, J., \& Stein, B. (1986). Solución IDEAL de problemas. Barcelona: Labor.

Cabero, J. (2015). Reflexiones educativas sobre las tecnologías de la información y la comunicación (TIC). Tecnología, Ciencia y Educación, I, 19-27.

Calle-Álvarez, G.Y. (2013). Lineamientos teóricos y didácticos para la construcción de ambientes de aprendizaje apoyados por TIC que favorezcan habilidades del pensamiento crítico. Medellín: Memorias XIV Encuentro Internacional Virtual
Educa. Recuperado de http://www.virtualeduca.info/fveduca/es/tematica-2013/94-la-escuela-de-la-era-digital/72I-lineamientos-teoricos-y-didacticos-para-la-construccionde-ambientes-de-aprendizaje-apoyados-por-tic-que-fortalezcan-las-habilidades-del-pensamiento-critico.

Calzadilla, M. (2002). Aprendizaje colaborativo y tecnologías de la información y la comunicación. Revista lberoamericana de Educación, 29(I), I-10.

Coronel, M., \& Curoto, M. (2008). La resolución de problemas como estrategia de enseñanza y aprendizaje. Revista Electrónica de Enseñanza de las Ciencias, 7(2), 463-479.

Díaz-Barriga, F. (2008). Educación y nuevas tecnologías de la información: ¿hacia un paradigma educativo innovador? Sinéctica, Revista Electrónica de Educación, (30), I-I5.

Echazarreta, C., Prados, F., Poch, J., \& Soler, J. (2009). La competencia "El trabajo colaborativo": una oportunidad para incorporar las TIC en la didáctica universitaria. Descripción de la experiencia con la plataforma ACME (UdG). UOC Papers: Revista sobre la Sociedad del Conocimiento, (8), 3.

Escudero, C. (2009). Una mirada alternativa acerca del residuo cognitivo cuando se introducen nuevas tecnologías. El caso de la resolución de problemas en ciencias. Revista Electrónica Teoría de la Educación. Educación y Cultura en la Sociedad de la Información, I0(I), 272-292.

Evans, E. (2010). Orientaciones metodológicas para la investigación-acción. Ministerio de Educación del Perú. Lima-Perú.

García, A. (1988). Evaluación de estrategias de resolución de problemas. Revista de Educación, (287), 275-286.

Gutiérrez, P., Yuste, R., Delgado, S., \& Fustes, M. (20I I). Buenas prácticas en el desarrollo de trabajo colaborativo en materias TIC aplicadas a la educación. Profesorado. Revista de Currículum y Formación de Profesorado, I5(I), 179-194.

Gutiérrez-Rodríguez, C. A. (2018). Fortalecimiento de las competencias de interpretación y solución de problemas mediante un entorno virtual de aprendizaje. Revista de Investigación, Desarrollo e Innovación, 8(2), 279-293. http:// dx.doi.org/I 0.19053/20278306.v8.n2.2018.7I 70

Maldonado, M. (2008). Aprendizaje basado en proyectos colaborativos. Una experiencia en educación superior. Laurus, 14(28), 158-180.

Marchesi, A., \& Díaz, T. (2009). Desafíos de las TIC. El cambio educativo en Iberoamérica. Telos: Cuadernos de Comunicación e Innovación, (78), I I I-I I 4.

McKernan, J. (1999). Investigación-acción y currículum: métodos y recursos para profesionales reflexivos. Ediciones Morata. 
Ministerio de Educación Nacional. (2008). Orientaciones generales para la educación en tecnología. Ser competente en tecnología: juna necesidad para el desarrollo!

Morales, P., \& Landa,V. (2004). Aprendizaje basado en problemas. Theoria, I3(I), 145-I57..

Moreno-Martínez, N. M., López-Meneses, E., \& Leiva-Olivencia, J.J. (2018). El uso de las tecnologías emergentes como recursos didácticos en ámbitos educativos. International Studies on Law and Education, 29(30), I31-146.

Navarro, S. (2006). La resolución de problemas planteada como un proceso de investigación hacia la enseñanza de la física. Prospectiva, 4(2), 60-65.

OCDE. (2017). Resultados PISA 2015 (vol.V): resolución colaborativa de problemas, PISA. Publicaciones de la OCDE, París. http://dx.doi.org/ I0.1787/978926428552I-en

Peralta, J. (20|8).Técnicas para la enseñanza de resolución de problemas. Revista Electrónica en Ciencias Sociales y Humanidades Apoyadas por Tecnologías, 7(I), 26-32.

Poblete, M., \& Villa, A. (2007). Aprendizaje basado en competencias. Una propuesta para la evaluación de competencias genéricas. Bilbao: Mensajero.

Polya, G. (1989). Cómo plantear y resolver problemas. México: Trilla.

Pozner, P., \& Fernández, T. (2000). Resolución de problemas. En Instituto Internacional de Planeamiento de la Educación. Diez módulos destinados a los responsables de los procesos de transformación educativa. Buenos Aires. Recuperado de http://poznerpilar.org/biblioteca/museo_feria/www.pgi. me.gov.ar/download/Mod07.pdf.

Pozo, J., \& Postigo, Y. (1994). La solución de problemas como contenido procedimental de la educación obligatoria. En J. Pozo, M. Pérez, J. Domínguez, M. Gómez y Y. Postigo. La solución de problemas. Madrid: Santillana.
Revelo-Sánchez, O., Collazos-Ordóñez, C., \& Jiménez-Toledo, J. (20 I8). El trabajo colaborativo como estrategia didáctica para la enseñanza/aprendizaje de la programación: una revisión sistemática de literatura. TecnoLógicas, 2 I(4I), II5-134.

Rodríguez, D., \& Valldeoriola, J. (20I2). Metodología de la investigación. México: Red Tercer Milenio.

Serrano, J., Cristancho, R., \& Soler, M. (2016). Estudio de estructuras artificiales: actividad tecnológica escolar por resolución de problemas y alineamiento constructivo. Revista Iberoamericana de Educación en Tecnología y Tecnología en Educación, 18, 60-70.

Solano, M. (20I2). Resolución de problemas de combinatoria en una wiki (tesis de maestría). Medellín: Facultad de Educación, Universidad de Antioquia. Recuperado de http://ayura.udea.edu.co:8080/jspui/bitstream/I23456789/169/1/ JH0769.pdf.

Tejedor-Tejedor, F. J. (2018). Investigación educativa: la utilidad como criterio social de calidad. Revista de Investigación Educativa, 36(2), 315-330. http://dx.doi.org//0.6018/ rie.36.2.3263 I I

Torrecilla, F. (20I I). Métodos de investigación en educación especial. Madrid: Universidad Autónoma de Madrid.

Travieso-Valdés, D., \& Ortiz-Cárdenas, T. (2018). Aprendizaje basado en problemas y enseñanza por proyectos: alternativas diferentes para enseñar. Revista Cubana de Educación Superior, 37(I), I24-133.

Vaca,J.,Agudo, J., \& Sánchez, H. (20|4). Evaluación de prácticas de programación mediante rúbricas en Moodle. Jornadas de Enseñanza Universitaria de la Informática. Oviedo. Recuperado de http://upcommons.upc.edu/bitstream/handle/2099/I5458/PI07va_eval.pdf. 